\title{
TEMPERATURE D'PENDENT VISCOUS DRAG IN CLOSE-PACKED METALS
}

\author{
T. Vreeland, Jr. and K. M. Jassby \\ W. M. Keck Lıboratory of Engineering Materials \\ California Institute of Technology, Pasadena, California 91109
}

\begin{abstract}
The influence of viscous drag on dislocation motion in close-packed metals is examined. Three experimental measurements of the viscous drag are discussed, i. e. internal friction, strain rate vs stress, and stress-time-displacement measurements. Experimental results of each of these methods are compared. Theories of dislocation-phonon and dis1ocation-electron interactions leading to viscous drag are briefly described. The experimentally-determined dislocation drag coefficient is qualitatively in agreement with the predictions of damping through dislocationphonon interactions. It is concluded that additional theoretical work is needed for a quantitative comparison of theory and experiment. Additional experimental work to determine the temperature dependence of the drag coefficient below $66^{\circ} \mathrm{K}$ is needed to resolve discrepancies in different theories of the dislocation-electron interaction.
\end{abstract}




\section{INTRODUCTION}

Viscous drag on dislocations implies the existence of a dissipative force, proportional to dislocation velocity. The consequences of such a force and measurement of its magnitude are treated in this paper, and theories of its origin are briefly described. We will restrict our attention to dislocations whose displacement does not involve the generation and motion of abrupt kinks, as exemplified by dislocations in closepacked metals.

It will be shown that the existence of a viscous drag force has a profound effect on the slip behavior of crystalline solids even under conditions where the viscous forces on dislocations do not dictate the stress levels required to sustain the deformation rate. As dislocation velocities increase, the viscous drag forces on dislocations predominate over the other forces which act to resist dislocation motion, and under this condition the magnitude of the viscous drag and the number of moving dislocations dictate the stress level required to sustain the deformation rate.

\section{DISLOCATION MOTION WITH LOCALIZED OBSTACLES AND VISCOUS DRAG}

Frost and Ashby ${ }^{1}$ have analyzed the motion of a dislocation passing through an array of discreet obstacles and acted upon by a viscous drag. Their calculations predict that applied stresses of the order of twice the critical stress to break through the obstacles will produce essentially the free-flight dislocation velocity which is governed by the viscous drag alone (and not the obstacles). At lower applied stresses, both the viscous drag and the obstacles influence the average dislocation velocity. The dislocation motion which may be steady on a macroscopic 
scale, is then discontinuous o' a scale which resolves the obstacles. If the obstacles are strong tut thermally penetrable, the average dislocation velocities at low stresses will be governed by the properties of the obstacles (not the viscous drag). The time for thermal activation is then long compared to the transit time between obstacles. The viscous drag plays an important role even in this case, as it limits the kinetic energy of the dislocation to a small fraction of the work done by the applied stress in moving the dislocation from obstacle to obstacle. If the viscous drag were absent, this work would go into kinetic energy and be available to overcome the next obstacle. Thus, the obstacles would not control the dislocation velocity if there were no viscous drag.

To illustrate how effectively a small viscous drag dissipates the work done in driving a dislocation from obstacle to obstacle we will consider the simplified case of a straight dislocation, initially at rest but free to move under the influence of the applied stress and a viscous drag. The equation of motion for a unit length of dislocation is then

$$
m \frac{d v}{d t}+B v=\tau b
$$

$$
\begin{aligned}
& \text { where } \quad m=\text { mass per unit length of dislocation } \\
& \mathrm{v}=\text { velocity and } \mathrm{v}=0 \text { at time } \mathrm{t}=0 \text {, } \\
& B=\text { viscous drag coefficient, } \\
& T=\text { applied resolved shear stress, and } \\
& b=\text { magnitude of the Burgers vector. }
\end{aligned}
$$

The solution of Eq. 1 is 


$$
v=v_{t}\left(1-e^{-\frac{B}{m} t}\right)
$$

where $v_{t}=T b / B$ is the terminal velocity. The dislocation velocity approaches the terminal velocity with an acceleration time constant $\frac{m}{B}$. The displacement is given by

$$
s=v_{t}\left[t-\frac{m}{B}\left(1-e^{-\frac{B}{m} t}\right)\right] \text {. }
$$

The distance moved before the dislocation achieves $90 \%$ of its terminal velocity is given by

$$
\mathrm{s}_{90}=1.4 \tau \mathrm{\tau m} / \mathrm{B}^{2} \text {. }
$$

This distance increases with stress and for non-relativistic velocities ( $v<c / 10$ where $c$ is the appropriate sonic velocity)

$$
\mathrm{s}_{90}<0.14 \mathrm{~cm} / \mathrm{B} \text {. }
$$

Taking $\mathrm{m}=\mathrm{m}_{\mathrm{o}} \mathrm{b}^{2}$ where $\mathrm{m}_{\mathrm{o}}=$ mass per unit volume of the crystal, and the experimental value for $B$ at room temperature for aluminum (discussed below) gives

$$
s_{90}<10 b .
$$

Thus the dislocation reaches $90 \%$ of its terminal velocity before it travels a distance of $10 \mathrm{~b}$. This estimate of an upperbound for $\mathbf{s}_{90}$ should hold for a11 close-packed metals. From this we conclude that for obstacle spacings large compared to $10 \mathrm{~b}$ the dislocation moves most of the way between obstacles at essentially its terminal velocity with the viscous drag force essentially equal to the driving force. The work done by the 
applied stress in moving the 'islocation from obstacle to obstacle is essentially all dissipated. Without a viscous drag this work would go into kinetic energy which would be available to overcome the next obstacle, and a steady state velocity $\left(v_{t} \ll c\right)$ would not occur. In this way the viscous drag force plays an important role, even under conditions where the stress level required to maintain the deformation rate is governed by the strength of the obstacle rather than by the magnitude of the viscous drag coefficient.

When internal stresses are present with a wave length much greater than $10 \mathrm{~b}$, the dislocation will travel with a varying velocity which is at any point essentially equal to the terminal velocity associated with the stress at that point. $\mathrm{Li}^{2}$ has shown that the average velocity of a dislocation is decreased by such an internal stress, even when the internal stress has a mean value equal to zero. The energy dissipated by viscous drag is unaffected by the internal stress when the mean value of the internal stress over the path of motion is zero.

\section{MEASUREMENT OF THE VISCOUS DRAG COEFFICIENT.}

Three different experimental methods have been used to obtain estimates of the viscous drag coefficient in close-packed metals. They are internal friction measurements, stress vs strain rate measurements at strain rates $>10^{2} \mathrm{sec}^{-1}$, and dislocation displacement measurements.

The internal friction method employs very low alternating stress levels such that the dislocation network is vibrated, usually without breaking, away from pinning points. Energy is dissipated by the viscous drag on the vibrating dislocations resulting in a decay in the amplitude of the alternating stress. 
Measurement of the strain and strain rate at stress levels where dislocation velocity is controlled by the viscous drag (and not other obstacles) gives a measure of the viscous drag if the density of moving dislocations is known. Measurement of dislocation displacement produced by a stress pulse of known duration and amplitude permits calculations of the viscous drag coefficient when the stress amplitude is sufficiently large to produce viscous drag-controlled velocities. Results of experimental measurements of $B$ using each of these three methods are discussed below.

\section{Internal Friction Measurements}

The first indirect measurements of $B$ were obtained from internal friction experiments. The experiments were interpreted on the basis of the vibrating string mode $1^{3}$. A recent review of internal friction and elastic modulus measurements is given in reference 4. In contrast with the other methods of measuring $B$, the dislocation velocities in the internal friction experiments are less than $10 \mathrm{~cm} / \mathrm{sec}$, and the dislocation displacements are usually only a few b. Four techniques which do not require knowledge of the spacing or distribution of pinning points along the dislocations have been employed to deduce $B$ from internal friction measurements:

1) Measurement of the decrement vs frequency (at constant temperature) around the damped resonant peak.

2) Determination of the asymptotic bchavior of the decrement (at constant lomperature) at frequencies above the damped resonant peak.

3) Measurement of the decrement vs temperature at constant frequency around the decrement peak. 
4) Measurement at two frecuencies of the stress amplitude which produces an internal friction peak in a specimen in both the superconducting and the normal state.

Methods 1) and 2) require the assumption that the dislocation network lengths do not change with frequency, while method 3) requires that these lengths do not change with temperature. The first three methods give the ratio of $B$ to the density of dislocations that contributes to the damping. Method 4) obtains B without any assumption about the dislocation density, but it is based upon a more complicated dislocation model (methods 1-3 apply to the low stress range where the decrement is independent of stress amplitude).

The values of B calculated from decrement measurements will be overestimated when energy dissipation by processes other than viscous drag on dislocations takes place. Alers and Salama ${ }^{5}$ measured the dislocation contribution to the elastic modulus and to the decrement in Cu (by observing these quantities before and after irradiating the specimen to tie up the dislocations). Their measurements are shown in Figs. 1 and 2 . The decrement measurements, Fig. 1, indicate that the dislocation contribution (the difference between the two measurements) is very sma11 at low temperatures and increases markedly above about $20^{\circ} \mathrm{K}$. The decrement in both irradiated and unirradiated material rose rapidly below $20^{\circ} \mathrm{K}$. The frequency used in this experiment was well above the resonant frequency for the unpinned dislocation network, so the difference in the two curves is essentially proportional to B. The dislocation contribution to the shear modulus, Fig. 2, was found to be a maximum near $0{ }^{\circ} \mathrm{K}$ and to decrease with an incroase ln tomperature up to $300^{\circ} \mathrm{K}$. This behavior is to be expected if 
$B$ is small at low temperature aid increases with increasing temperature. Alers and Thompson ${ }^{6}$ were the first to report values of $B$ for single crystals of copper measured by internal friction techniques. Their estimates were somewhat higher than those found in the direct measurements discussed below, but subsıquent measurements by Thompson and Young ${ }^{7}$ (using refined determinations of the dislocation density) have brought the internal friction and direct measurements into agreement (in the temperature range $\left.66^{\circ}-300^{\circ} \mathrm{K}\right)$.

Mason ${ }^{8}$ has reported $B$ values obtained from internal friction measurements in aluminum and copper. His measurements, Fig. 3, show a decrease in $\mathrm{B}$ with a decrease in temperature down to about $100^{\circ} \mathrm{K}$, and a rapid rise in $\mathrm{B}$ with decreasing temperatures below $100^{\circ} \mathrm{K}$. The low temperature behavior is in contrast to the results of Alers and Thompson ${ }^{6}$ and Alers and Salama ${ }^{5}$. Seeger ${ }^{9}$ has pointed out that a large B as reported by Mason at low temperatures would essentially prevent the observed dislocation contribution to the modulus decrement at the high frequencies. To counter this, Mason ${ }^{9}$ has argued that the low temperature measurements may be affected by a temperature dependence of the dislocation loop lengths. However, Granato $^{4}$ explained that, at high frequencies, the effect of pinning points becomes negligible and then the temperature dependence of loop lengths does not effect the temperature-dependence of $B$.

Hikata and Elbaum ${ }^{10}$ used measurements of the shift in amplitude at maximum ultrasonic attenuation in normal and superconducting lead to determlne $B$ fn the normal state. They found at $4.2^{\circ} \mathrm{K}$ and $11^{\circ} \mathrm{K}$, $B=8 . \times 10^{-5}$ dyne sec $\mathrm{cm}^{-2}$ (independent of any ad hoc assumptions about dislocation density or loop length). The validity of this measurement 
is in question however, since the model for breakaway from pinning points which was used in their analysis is applicable only to low frequency stressing, while the experiments were carried out at 10 and $2 \mathrm{MHH}_{z}$.

\section{High Strain Rate Experiments}

Indirect measurements of the drag coefficient have been obtained from the applied stress vs strain rate behavior at high strain rates $\left(10^{2}-10^{5} \mathrm{sec}^{-1}\right)^{11-17}$. A 1 inear relationship between stress and strain rate has been observed and this implies that a viscous drag acts on dislocations if the moving dislocation density is independent of stress. The high stresses employed in this method usually produce dislocation velocities in excess of $10^{3} \mathrm{~cm} / \mathrm{sec}$.

Ferguson, Kumar and Dorn ${ }^{12}$ found a linear relationship between stress and strain rate in single crystals of aluminum. Their experimental data is represented by an equation of the form

$$
\tau-\tau_{0}=\alpha \dot{\gamma}
$$

where

$$
\begin{aligned}
\tau_{0} & =\text { constant } \\
\alpha & =\text { temperature-dependent constant } \\
\dot{\gamma} & =\text { strain rate } .
\end{aligned}
$$

They assume that the dislocations are moving at the terminal velocity

$$
v_{t}=\left(\tau-\tau_{0}\right) b / B
$$

so that

$$
\dot{\gamma}=\rho b v_{t}=\rho b \dot{L}\left(1-\tau_{0}\right) / B
$$

where $p=$ density of the moving dislocations. Comparison of equations 
7 and 9 indicates that $\rho$ is $j$ ndependent of stress and that

$$
B=\alpha p b^{2}
$$

While the use of an effective stress ( $\left.\tau-\tau_{0}\right)$ in Eq. 8 may not be strictly $\operatorname{valid}^{18}$, these experimentai values of $\alpha$, and an estimate of $\rho$ permit the calculation of B. Using this method the drag coefficient was found to decrease slightly with increasing temperature in the range $20-500^{\circ} \mathrm{K}$. Alternately, Eq. 10 may be used to calculate $\rho$ in the high strain rate experiments using the experimental value of $\alpha$, and the appropriate $B$ determined from more direct measurements. The data of Ferguson et al would indicate that $\rho 300^{\circ} \mathrm{K} / \rho_{70} \mathrm{~K}$ is approximately 3 using Eq. 10 and the $\mathrm{B}$ values of Gorman, Wood and Vreeland ${ }^{19}$.

Yoshida and Nagata have reported high strain rate measurements in single crystals of $\mathrm{Cu}^{14}, \mathrm{Cu}-\mathrm{Mn}$ alloys ${ }^{15}$, and $\mathrm{Zn}^{16}$. The data for $\mathrm{Cu}$ obeyed Eq. 7 with $\tau_{0}=0$, and using the initial dislocation density in Eq. 10 they found $\mathrm{B}=1.3 \times 10^{-4}$ dynes $\mathrm{sec} \mathrm{cm}^{-2}$ at $293^{\circ} \mathrm{K}$. Further, they reported the following qualitative temperature dependence: B decreased with decreasing temperature below $293^{\circ} \mathrm{K}$ in $\mathrm{Cu}$ and $\mathrm{Cu}-0.1 \% \mathrm{Mn}$ while the opposite was found in the $0.5 \%$ and $1.1 \%$ Mn alloys. Their value of the viscous drag coefficient at room temperature agrees remarkably well with the value determined by the more direct method reported below. Yoshida and Nagata did not report values of B for zinc, but their measurements show that the yield point for basal slip, at a strain rate of $7 \times 10^{2} \mathrm{sec}^{-1}$, dropped from $260 \mathrm{~g} / \mathrm{mm}^{2}$ at $300^{\circ} \mathrm{K}$ to about $200 \mathrm{r} / \mathrm{mm}^{2}$ at $196^{\circ} \mathrm{K}$. This implies that $\mathrm{B} 300^{\circ} \mathrm{K} / \mathrm{B} 196^{\circ} \mathrm{K}=260 / 200$ $=1.3$ (assumfing $\rho=$ constant and ${ }_{0}=0$ ). The direct measurcments of B for basal dislocations over this temperature range gave the same ratio 
(Pope and Vreeland ${ }^{20}$ ).

\section{Direct Measurements}

The most direct measurement of $\mathrm{B}$ which is presently practical involves the determination of the time duration of stress and the stress amplitude required to produce a measurable dislocation displacement. These measurements can be related to B most simply when both the critical stress to break obstacles and the internal stresses are negligible compared to the applied stress. Equation 3 is then applicable and for $\mathrm{s}>>\mathrm{s}_{90}$

$$
\mathrm{B}=\tau \mathrm{bt} / \mathrm{s} \text {. }
$$

It is possible to study the dependence of $B$ on dislocation orientation by this method. However, the direct measurements become impractical when dislocation densities are high, and are increasingly difficult at dislocation velocities approaching the sonic velocity. When internal stresses or obstacle strengths are not small compared to the applied stress, dislocation displacements are reduced and $\mathrm{B}$ is overestimated.

Techniques for the direct measurement of dislocation mobility are discussed in reference 21. Selective introduction of dislocations in crystals containing very low dislocation densities, their observation by the Berg-Barrett $\mathrm{x}$-ray technique, and the use of short duration (10-10au sec) torsional stress pulses to produce measureable displacements have proved to be effective in giving a direct measure of $\mathrm{B}$ in $\mathrm{Zn}^{20}, \mathrm{Al}^{19}$ and $\mathrm{Cu}^{22}$. An example of dislocation displacements produced by a torsional stress pulse applied to a cylindrical crystal of zinc is shown in Fig. 4. The two nearly radial scratches seen on the basal surface, one continuous and 
one interrupted, were made prior to the application of the stress pulse. The scratching produced long edge dislocations close to and parallel with the scratch. The dislocations at the center of the bottom edge of Fig. 4 (the axis of the cylindrical specimen) are representative of the initial configuration of all the dislocations after scratching. The torsional stress pulse produced displacements which are clearly linear with radius on the continuous scratch. The interrupted scratch shows the effect of local "obstructions." As the torsional stresses vary linearly with radius, and the entire surface experienced the same duration of loading, we obtain the viscous drag coefficient from Equation 10. Dislocation velocities in excess of $10^{4} \mathrm{~cm} / \mathrm{sec}$ have been produced by this method with displacements up to about $1 \mathrm{~mm}$.

Measured values of $B$ for edge dislocations are shown in Fig. 5 as a function of temperature. The $B$ values increase with increasing temperature between $66^{\circ}$ and $100^{\circ} \mathrm{K}$ which is in contrast with Mason's results ${ }^{8}$. Pope and Vreeland ${ }^{20}$ measured $B$ as a function of dislocation orientation for basal dislocations in $\mathrm{Zn}$. They found that B for edge oriented dislocations was about $25 \%$ greater than B for screw oriented dislocations, while the temperature dependencies were similar.

\section{THEORIES OF THE VISCOUS DRAG}

Theories of dislocation interaction with phonons and with conduction electrons have been proposed which predict viscous drag. Three distinct phonon interaction mechanisms have been discussed in the literature: Phonon interaction with the moving strain field of the dislocation, anharmonic radiation from the dislocation core, and phonon induced 
flutter of the dislocation line.

Three physical processes nave been put forward to describe the interaction of thermal phonons with the strain field of the moving dislocation. These include the thermoelastic effect, the phonon viscosity effect, and the phonon scattering mechanism. The thermoelastic and viscosity effects can be shown to be two separate parts of the same thermal phonon-strain field interaction, first proposed by Akhieser ${ }^{23}$. Akhieser clearly demonstrated the lattice absorption of energy from strain waves with long wavelengths relative to the thermal phonon mean free path. He reasoned that the thermal phonons are highly localized with respect to the varying strain field and can be considered as traveling through a uniform medium with a slowly modulating strain field. The lattice thermal frequencies are changed as the result of the impression of the strain, creating temperature differences between phonon modes at any spatial point. The temperature differences themselves are spatially-dependent, according to the spatial dependence of the strain field. A two step relaxation process re-establishes thermal equilibrium in the crystal.

Firstly, the phonons at any point relax to a new thermal equilibrium at some common temperature (adiabatic temperature) through phonon-phonon collisions. Return to the general isothermal condition is then governed by the macroscopic parameters involved in heat flow between spatial points. The energy absorbed from a moving dislocation strain field in the latter stage (adiabatic-isothermal transition) has been determined exactly by Eshelby ${ }^{24}$ and Weiner ${ }^{25}$ and is negligibly small for metals.

The former step has been considered by Mason and others ${ }^{26-28}$, who presented approximate calculations for the energy absorption. However, difficulties arise in consideration of the region of the dislocation 
strain field that can interact with thermal phonons and in determination of appropriate phonon relaxation times.

The phonon scattering mechanism considers the scattering of thermal phonons by the strain field of a moving dislocation and the difference in crystal momentum imparted to phonons in the forward and back-scattered directions. These mechanisms have been reviewed by Lothe ${ }^{29}$. It has not yet been clearly demonstrated whether the scattering and viscosity mechanisms are indeed different mathematical approaches to the same problem or apply to different regions of the phonon spectrum.

It is not possible, at this date, to closely approximate the experimental determination of the drag coefficients of several soft metals (Fig. 5) by one or a combination of phonon mechanisms over a wide temperature range.

Electron Interaction with the Moving Strain Field

Conduction electrons interacting with a moving dislocation give rise to a viscous drag. This interaction may become dominant at low temperatures where the dislocation-phonon interaction is very weak. Mason ${ }^{30}$ proposed that the free electrons exert a viscous drag on the moving dislocation similar in effect to the phonon drag. The drag coefficient due to free electrons, according to Mason, is proportional to the electrical conductivity and therefore increases as the temperature decreases. The ultrasonic attenuation measurements of Mason and co-workers indicated an increasing $B$ with decreasing temperature. In the superconducting state the drag constant due to conduction electrons should decrease markedly.

V. Ya. Kravchenko ${ }^{31}$ treated the dislocation-electron interaction. 
He concluded that the drag coefficient was temperature independent. A similar conclusion was reached by Holstein, whose treatment appears in the appendix of a paper by Tittmann and Bormme ${ }^{32}$. Both Kravchenko and Holstein criticised Mason's treatment on the basis that it was based on an assumption which was grossly violated (the assumption that $q l \ll<1$ where $\ell=$ mean free path for conduction electrons and $\mathrm{q}=$ typical Fourier component of the dislocation strain field).

Huffman and Louat ${ }^{33}$ reconsidered the dislocation-electron interaction. Their treatment found $\mathrm{B}$ to be proportional to the electrical conductivity (Mason's result) although they considered $q l \gg>1$ in the spirit of the Kravchenko and Holstein treatments. No reconciliation of the conflicting conclusions regarding the behavior of $\mathrm{B}$ at low temperature has been made.

\section{SUMMARY AND CONCLUSIONS}

The existence of viscous drag on dislocations permits a quasisteady state dislocation motion to occur, with the work done by the applied forces being dissipated by the viscous forces. At high strain rates, the viscous forces on dislocations may exceed other sources of dislocation drag and the stress level is then directly proportional to the viscous drag coefficient. The temperature dependence of the stressstrain behavior will then be strongly influenced by the temperature dependence of the viscous drag coefficient.

Direct measurements demonstrate that the viscous drag coefficient increases with temperature in the range $66.300^{\circ} \mathrm{K}$ for dislocations on closepacked planess of $\mathrm{Zn}, \Lambda \mathrm{I}$ and Cu. Internal friction measurements ${ }^{7}$, stress vs strain ralo measurements ${ }^{14}$ and direct measurements ${ }^{22}$ in $\mathrm{Cu}$ all give cossentially the same value for $\mathrm{B}$ at $300^{\circ} \mathrm{K}$, and the same temperature dependence is indicated. The results of direct measurements below $66^{\circ} \mathrm{K}$ 
have not yet been published, and the two sources of indirect measurements in this range are in conflict with one another.

Above $66^{\circ} \mathrm{K}$, the mechanism for dislocation damping is recognized to be some form of dislocation-phonon interaction, although the experimental results do not correlate with one or a combination of mechanisms. It is concluded that more complete theories are required to allow a positive comparison of experimental and theoretical results.

Theories of the dislocation-electron interaction differ with regard to the predicted temperature dependence of $B$. Direct measurements at temperatures below $66^{\circ} \mathrm{K}$ are needed to resolve this controversy. 


\section{REFERENCES}

1 H. J. Frost and M. Ashby, Tech. Report No. 1, O.N.R. Contract N00014-67-A-0298-0020.

2 J. C. M. Li in Dislocation Dynamics, McGraw Hil1, New York, 1968, 87.

3 A. Granato and K. Lücke, J. App1. Phys., 27 (1956) 583.

4 A. Granato in Dislocation Dynamics, McGraw-Hi11, New York, 1968, 117.

5 G. A. Alers and K. Salama in Dislocation Dynamics, McGraw-Hill, New York, 1968, 211.

6 G. A. Alers and D. O. Thompson, J. App1. Phys., 32 (1961) 283.

7 D. O. Thompson and F. W. Young, Jr., Unpublished research.

8 W. P. Mason in Dislocation Dynamics, McGraw-Hil1, New York, 1968, 487.

9 A. Seeger discussion in Dislocation Dynamics, McGraw-Hi11, New York, 1968, 503.

10 A. Hikata and C. Elbaum, Phys. Rev. Letters, 18 (1967) 750.

11 F. E. Hauser, J. A. Simmons and J. E. Dorn in Response of Metals to

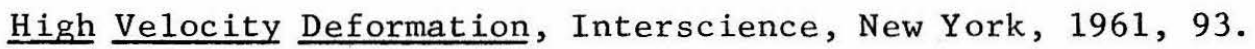

12 W. G. Ferguson, A. Kumar and J. E. Dorn, J. App1. Phys., 38 (1967) 1863.

13 A. Kumar, F. E. Hauser and J. E. Dorn, Acta Met. 16 (1968) 1189.

14 N. Nagata and S. Yoshida, J. Japan Inst. Meta1s, 31 (1967) 735 (in Japanese).

15 N. Nagata and S. Yoshida, J. Japan Inst. Metals, 32 (1968) 385 (in Japanese).

16 N. Nagata and S. Yoshida, Trans. Japan Inst. Metals, 9 (1968) 110.

17 A. Kumar and R. G. Kumble, J. App1. Phys. 40 (1969) 3475.

18 A. S. Argon, Mater. Sci. Eng., $3(1968 / 69) 24$.

19 J. A. Gorman, D. S. Wood and T. Vreeland, Jr., J. App1. Phys., 40 (1969) 833. 
20 D. P. Pope and T. Vreelanc, Jr., Phil. Mag., 20 (1969) 1163.

$21 \mathrm{~T}$. Vreeland, Jr. in Techniques for the Direct Observation of Structure and Imperfections, Interscience, New York, 1968, Vo1. II, Part I, p. 341 .

22 K. M. Jassby and T. Vreeland, Jr., Phil. Mag., 21 (1970) 1147.

23 A. Akhieser, J. Phys. (USSR), 1 (1939) 277.

24 J. D. Eshelby, Proc. Roy. Soc. (London), A 197 (1949) 396.

25 J. H. Weiner, J. App1. Phys., 29 (1958) 1305.

26 W. P. Mason, J. App1. Phys., 35 (1964) 2779.

27 W. P. Mason and T. B. Bateman, J. Ac. Soc. Am., 36 (1964) 644.

28 W. P. Mason and A. Rosenberg, J. Appl. Phys., 38 (1967) 1929.

29 J. Lothe, J. App1. Phys., 33 (1962) 2116.

30 W. P. Mason, App1. Phys. Letters, 6 (1965) 111.

31 V. Ya Kravchenko, Soviet Phys. - Solid State, 8 (1966) 740.

32 B. R. Tittmann and H. E. B'bmme1, Phys. Rev., 151 (1966) 178.

33 G. P. Huffman and N. P. Louat, Phys. Rev. Letters, 19 (1967) 518. 


\section{ACKNOWLEDGEMENTS}

This work was sponsored by the U. S. Atomic Energy Commission and the Division of Enginrering and Applied Science, California Institute of Technology. 


\section{FIGURF CAPTIONS}

Fig. 1 Temperature variatiou of the attenuation of a ten megacycle shear wave in an annealed copper single crystal before and after neutron irradiation (data of Alers and Salama ${ }^{5}$ ).

Fig. 2 Temperature variation of the elastic shear modulus $\mathrm{C}_{44}$ in annealed and neutron irradiated copper (data of Alers and Salama ${ }^{5}$ ).

Fig. 3 Temperature variation of the dislocation drag coefficient in aluminum and copper reported by Mason $^{8}$ (obtained from an analysis of internal friction measurements).

Fig. 4 A Berg-Barrett topograph of the basal surface of zinc taken after the application of a short duration torsion pulse at $66^{\circ} \mathrm{K}$. Note motion away from a continuous and an interrupted radial scratch (center of cylindrical specimen is at the left in the figure). 20.5X

Fig. 5 Temperature variation of the edge dislocation drag coefficient from direct measurements in $\operatorname{zinc}^{20}(0001)\langle 1 \overline{2} 10\rangle$, aluminum ${ }^{19}$ and copper ${ }^{22}$. 


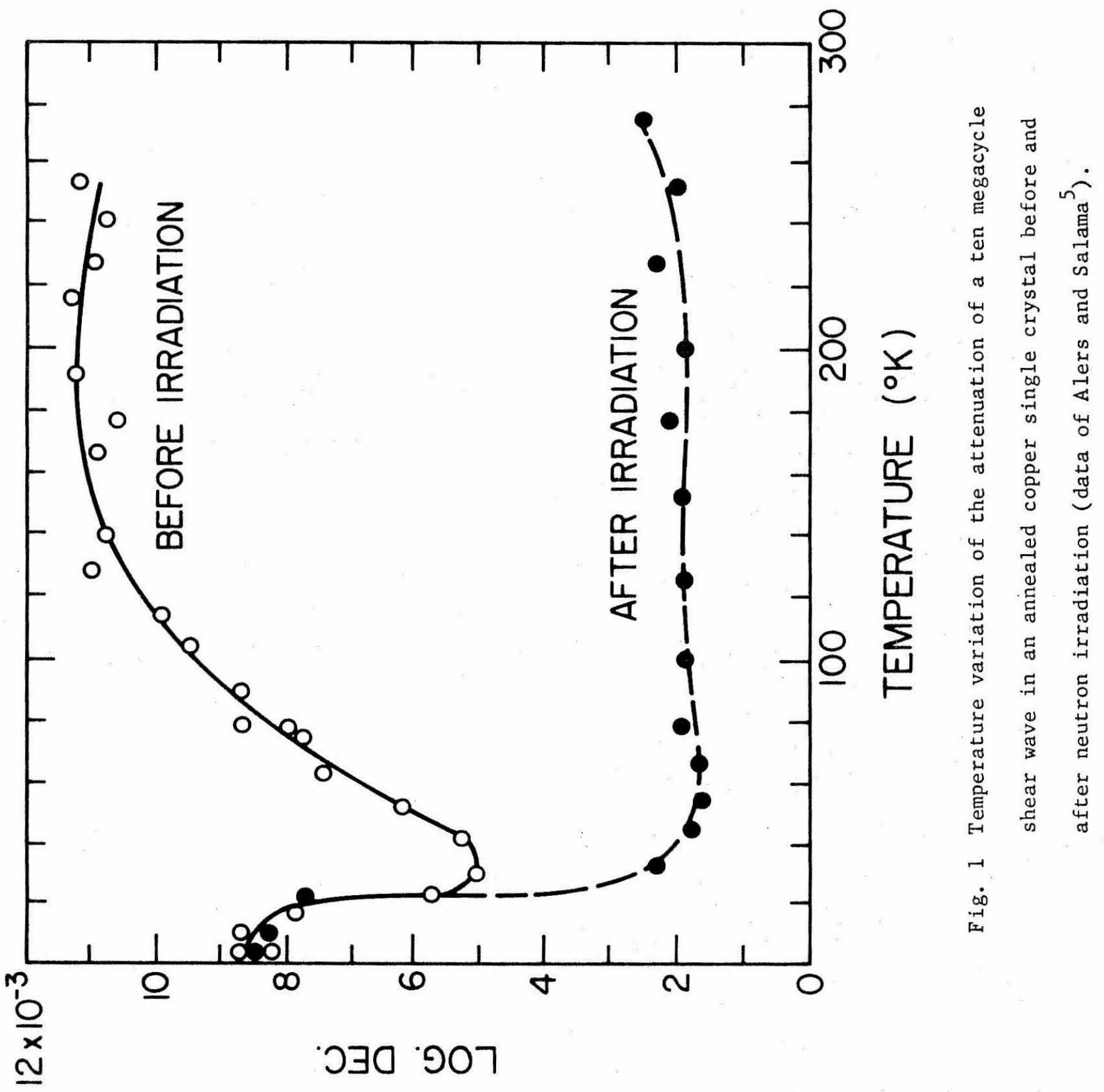




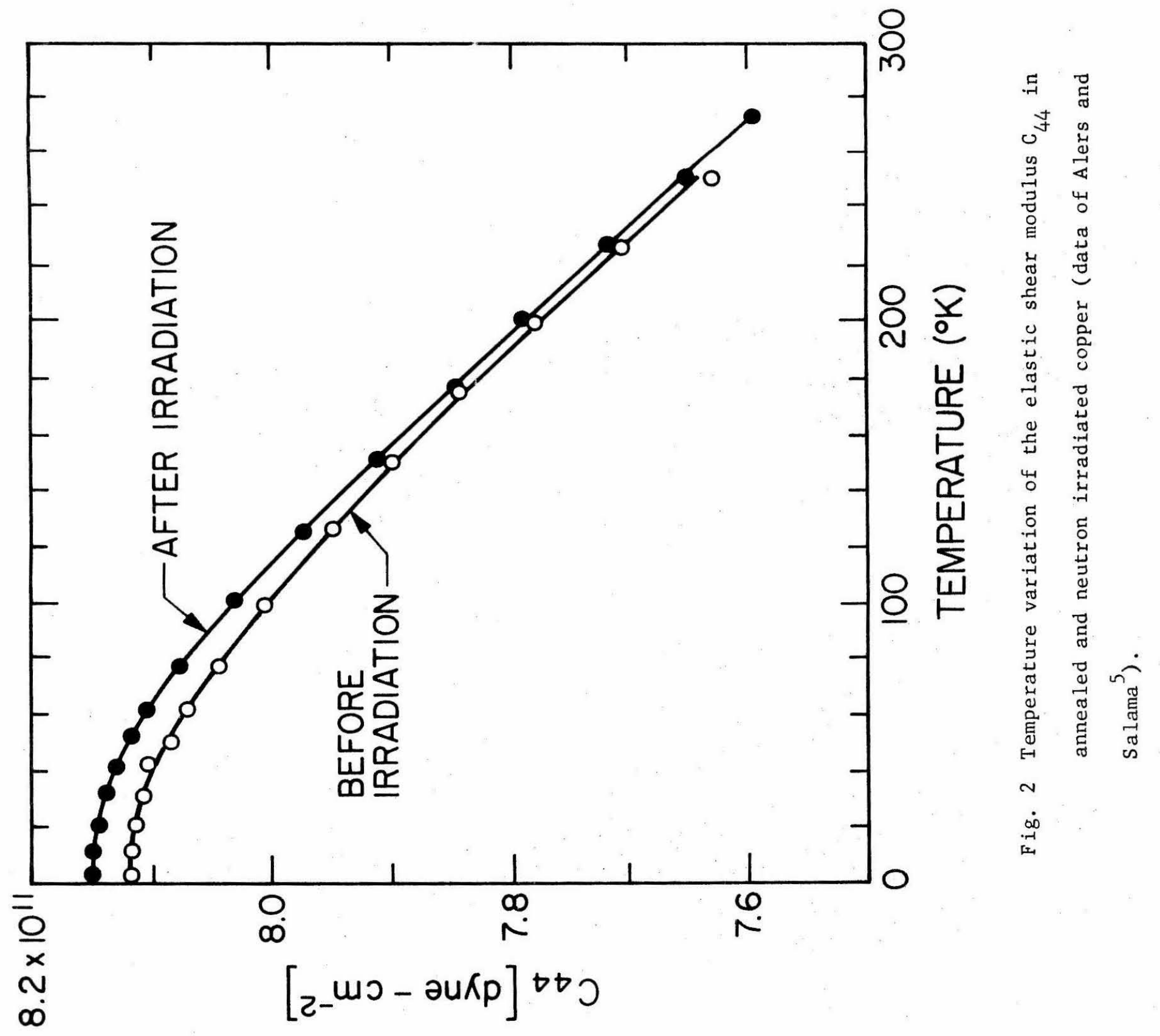




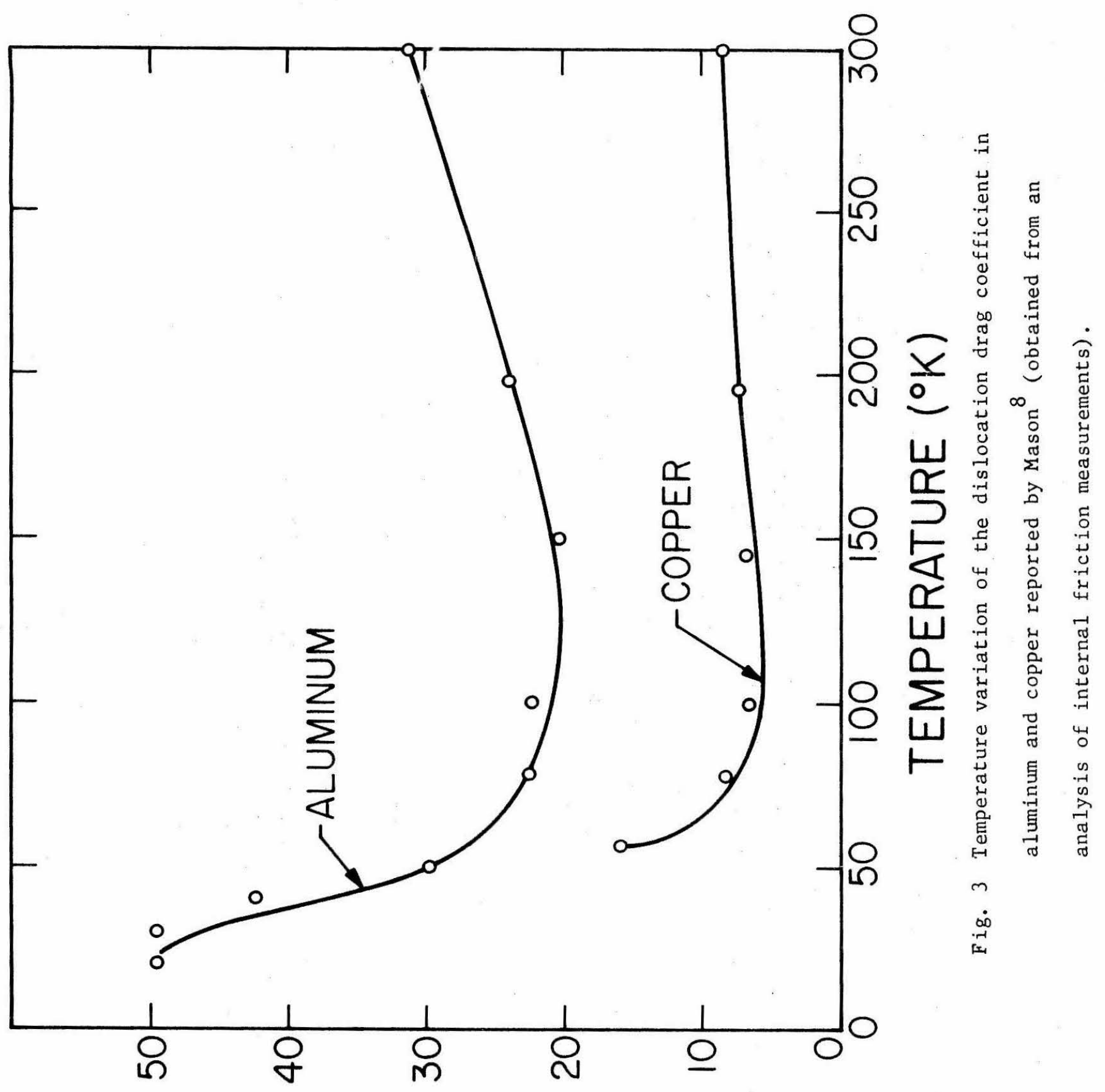

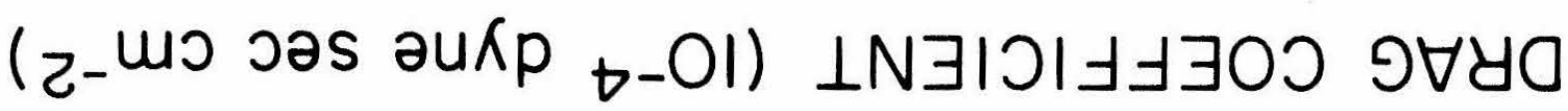




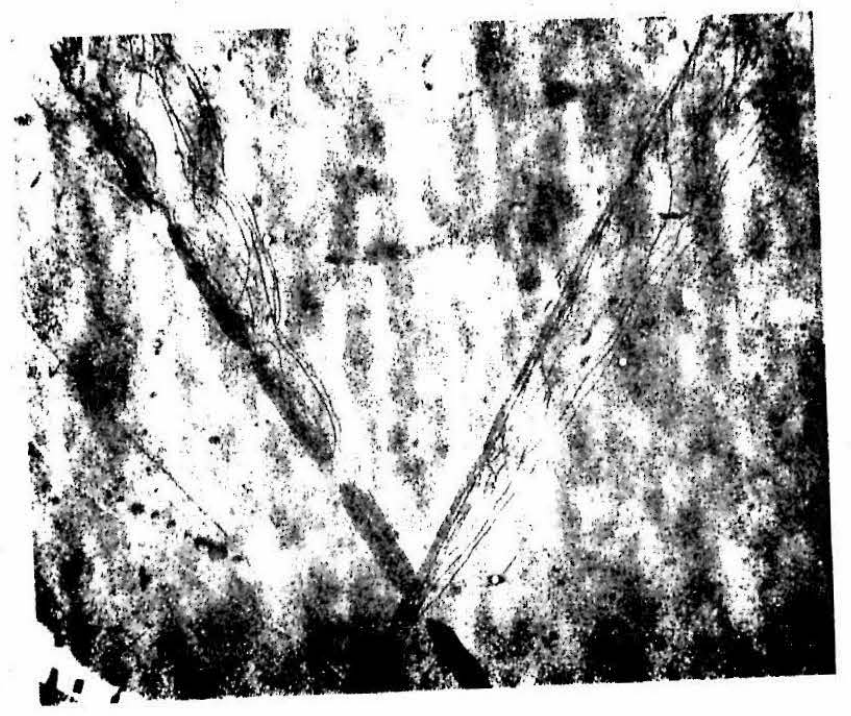

Fig. 4 A Berg-Barrett topograph of the basal surface of zinc taken after the application of a short duration torsion pulse at $66^{\circ} \mathrm{K}$. Note motion away from a continuous and an interrupted radial scratch (center of cylindrical specimen is at the left in the figure). 20.5X 


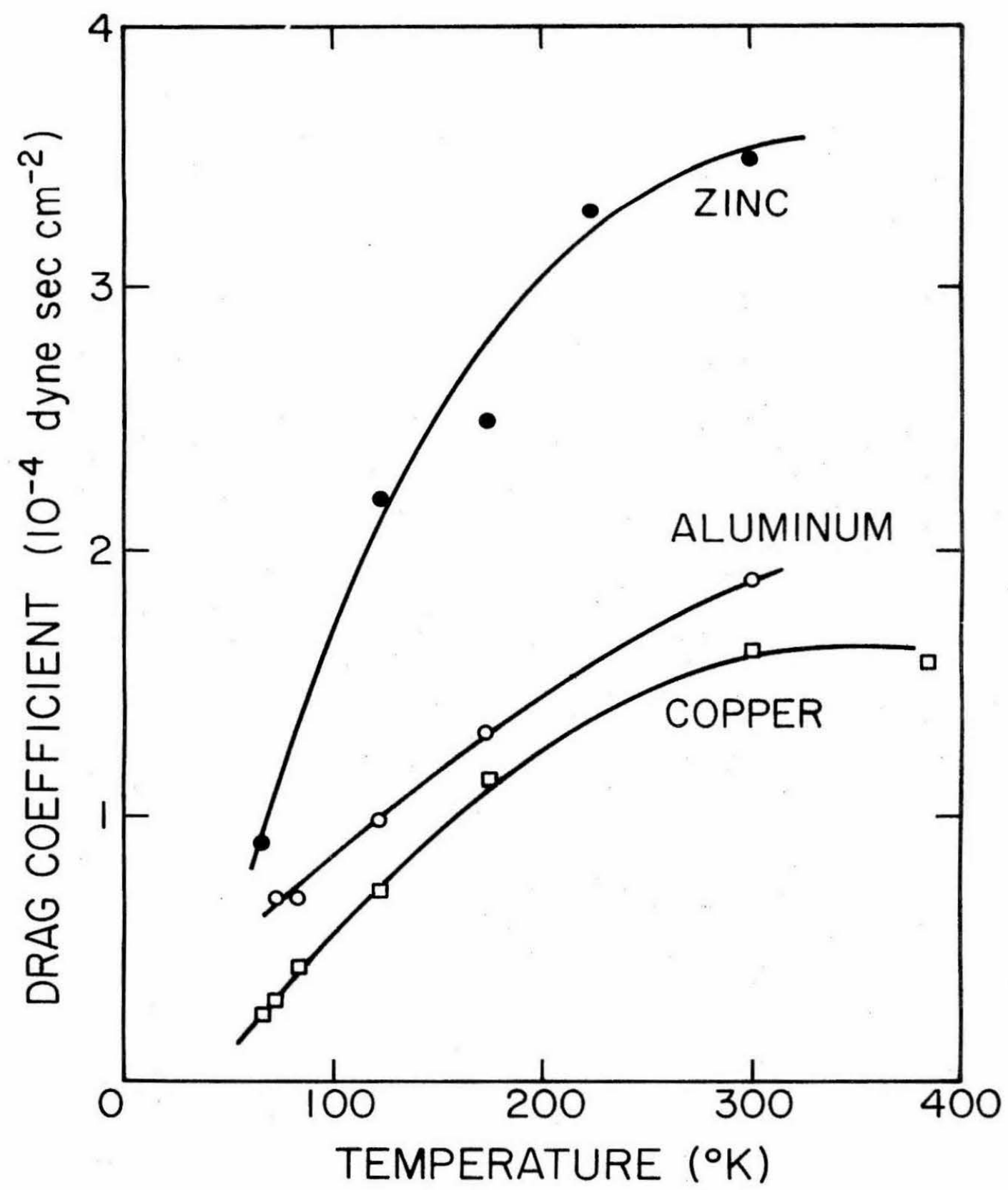

Fig. 5 Temperature variation of the edge dislocation drag coefficient from direct measurements in $\operatorname{zinc}^{20}(0001)\langle 1 \overline{2} 10\rangle$, aluminum 19 and copper 22 . 